\title{
Activation during Ventricular Defibrillation in Open-chest Dogs \\ Evidence of Complete Cessation and Regeneration of Ventricular Fibrillation after Unsuccessful Shocks
}

\author{
Peng-Sheng Chen, Nitaro Shibata, Ellen G. Dixon, Patrick D. Wolf, Ned D. Danieley, \\ Michael B. Sweeney, William M. Smith, and Raymond E. Ideker \\ Departments of Medicine and Pathology, Duke University Medical Center, Durham, North Carolina 27710
}

\begin{abstract}
To test the hypothesis that a defibrillation shock is unsuccessful because it fails to annihilate activation fronts within a critical mass of myocardium, we recorded epicardial and transmural activation in 11 open-chest dogs during electrically induced ventricular fibrillation (VF). Shocks of 1-30 J were delivered through defibrillation electrodes on the left ventricular apex and right atrium. Simultaneous recordings were made from septal, intramural, and epicardial electrodes in various combinations. Immediately after all 104 unsuccessful and 116 successful defibrillation shocks, an isoelectric interval much longer than that observed during preshock VF occurred. During this time no epicardial, septal, or intramural activations were observed. This isoelectric window averaged $64 \pm 22 \mathrm{~ms}$ after unsuccessful defibrillation and $339 \pm 292 \mathrm{~ms}$ after successful defibrillation $(P$ $<0.02$ ). After the isoelectric window of unsuccessful shocks, earliest activation was recorded from the base of the ventricles, which was the area farthest from the apical defibrillation electrode. Activation was synchronized for one or two cycles following unsuccessful shocks, after which VF regenerated.

Thus, $(a)$ after both successful and unsuccessful defibrillation with epicardial shocks of $\geq 1 \mathrm{~J}$, an isoelectric window occurs during which no activation fronts are present; (b) the postshock isoelectric window is shorter for unsuccessful than for successful defibrillation; (c) unsuccessful shocks transiently synchronize activation before fibrillation regenerates; $(d)$ activation leading to the regeneration of VF after the isoelectric window for unsuccessful shocks originates in areas away from the defibrillation electrodes. The isoelectric window does not support the hypothesis that defibrillation fails solely because activation fronts are not halted within a critical mass of myocardium. Rather, unsuccessful epicardial shocks of $\geq 1 \mathrm{~J}$ halt all activation fronts after which VF regenerates.
\end{abstract}

\section{Introduction}

Although much work has been done to determine the optimum electrodes, wave forms, and energy levels for defibrillation (1), little is known about the electrical effects of a defibrillation shock because it has not been possible to record simultaneously from many electrodes within the heart while a defibrillation shock is administered. Consequently, the mechanism of electrical defibrillation remains unclear. One possible mechanism is that successful defibrillation requires a shock that is strong enough to

Received for publication 29 April 1985 and in revised form 12 November 1985.

J. Clin. Invest.

(C) The American Society for Clinical Investigation, Inc.

0021-9738/86/03/0810/14 \$1.00

Volume 77, March 1986, 810-823 cause a period of prolonged unresponsiveness of myocardium (2), thought to be due to prolonged depolarization of the cell membrane by the shock (3). A second possible explanation is that the shock must simultaneously depolarize every excitable ventricular fiber so that all activation fronts are immediately annihilated $(4,5)$. The most commonly accepted explanation is that only the excitable fibers within a critical mass of myocardium must be depolarized (6); the shock increases the organization of cardiac electrical activity by decreasing the number of activation fronts present and by increasing the synchronism of the remaining activation fronts, so that VF cannot be perpetuated, and all activation fronts soon die out.

With the development of computer-assisted mapping systems (7), it has become possible to map activation during unstable arrhythmias (8). We have modified our mapping system to record soon after a countershock without amplifier saturation (9). The purpose of this study was to use this modified mapping system during ventricular fibrillation $(\mathrm{VF})^{1}$ to test the critical mass hypothesis of electrical defibrillation.

\section{Methods}

\section{Recording electrodes}

Signals were recorded digitally at a rate of 1,000 samples per second (10) from up to 64 electrodes simultaneously. Different arrays of electrodes were used in different parts of the study to record from the epicardium, from the septum, and from the intramural free wall. The study was divided into two parts, each performed in a different group of dogs according to a different protocol. In part I, we recorded global epicardial activation, regional epicardial activation, and septal activation after shocks delivered through two defibrillation electrodes. Results of these six dogs caused us to record right ventricular (RV) epicardial activation, intramural activation, and subendocardial activation in part II, with the same defibrillation electrode configuration.

Sock electrode arrays. Two sock electrode arrays (11) were used for epicardial recordings. In part I we used a sock that contained 56 bipolar button electrodes spaced evenly over the ventricular epicardium to obtain a global picture of epicardial activation. Each pole of the bipolar electrodes was $1 \mathrm{~mm}$ in diameter, and the poles were separated by $2.5 \mathrm{~mm}$ from center to center. A sock with 24 electrodes was used in part II to record the electrical activity over the RV outflow tract.

Plaque electrode array. A plaque electrode array was sutured directly to the epicardium in part I and recordings were made from 56 electrodes simultaneously to map in greater detail the spread of activation over a selected portion of the epicardium. The electrodes were within a trapezoidal silastic plaque, $10 \mathrm{~cm}$ long, $3.5 \mathrm{~cm}$ wide on one end, and $5 \mathrm{~cm}$ wide on the other. There were seven rows and eight columns of bipolar electrode pairs on the plaque; the distance between the poles of each bipole was $2.5 \mathrm{~mm}$. The bipolar electrode pairs were separated by 14 $\mathrm{mm}$ between the rows and by $7 \mathrm{~mm}$ between the columns.

Plunge electrode arrays. Two kinds of plunge electrodes were used in part I to record from the septum, and a third kind was used in part

1. Abbreviations used in this paper: LV, left ventricular, RV, right ventricular; VF, ventricular fibrillation; ww, window width. 
II to record through the RV free wall. One kind of plunge used in part I was $62 \mathrm{~mm}$ long and had two bipolar electrode pairs, $2.5 \mathrm{~mm}$ apart near the tip. The distance between the poles of each bipole was 0.75 $\mathrm{mm}$. The casing of the plunge was an 18-gauge intravenous catheter, with an anchor in the center which stabilized the electrode during the study and served as a marker for the electrode location during postmortem examination (12). Two of these plunges were inserted perpendicularly through the RV outflow tract and anchored at the left side of the interventricular septum to make septal recordings. The other plunge used was $29 \mathrm{~mm}$ long, and was constructed from a 21-gauge needle. It had four bipolar pairs separated by $0.5 \mathrm{~mm}$ from pole to pole with $2.5 \mathrm{~mm}$ between each bipolar electrode. It was inserted into the septum from the upper part of the interventricular sulcus. One plunge of this kind was used in each study. Thus a total of eight recordings from the three plunges was made from the septum in part $I$.

A third kind of plunge electrode was used for RV free-wall recordings in part II. These plunge electrodes were constructed from 21-gauge needles. Each had nine unipolar electrodes separated by $1 \mathrm{~mm}$. During the study, the neighboring unipolar electrodes were used to form eight bipolar pairs. The plunges were inserted at a $45^{\circ}$ angle into the $R V$ free wall, so that most of the electrodes on the plunge were within the RV free wall.

\section{Surgical preparation}

11 mongrel dogs (mean weight \pm standard deviation [SD], $19.3 \pm 2.2 \mathrm{~kg}$ ) were anesthetized with pentobarbital $(30-35 \mathrm{mg} / \mathrm{kg})(13,14)$ and succinylcholine $(1 \mathrm{mg} / \mathrm{kg})$. Each was intubated with a cuffed endotracheal tube and ventilated with $30-60 \%$ oxygen through a Harvard respirator (Harvard Apparatus Co., Inc., S. Natick, MA). Ringer's lactate was continuously infused and supplemented with potassium chloride, sodium bicarbonate, and calcium chloride when indicated. Via a separate intravenous line, pentobarbital was infused at a rate of $1 \mathrm{mg} / \mathrm{min}$ throughout the experiment to achieve adequate anesthesia. Succinylcholine at a bolus dose of $0.25-0.5 \mathrm{mg} / \mathrm{kg}$ was given no more than once per hour to decrease muscle contraction induced by the electric shock. This dose is much less than that required to cause significant changes of cardiac excitability (15). An arterial line was inserted into the femoral artery, and the systemic blood pressure was continuously displayed on an oscilloscope. Blood was withdrawn to determine the $\mathrm{pH}, \mathrm{PO}_{2}, \mathrm{PCO}_{2}, \mathrm{CO}_{2}$ content, bicarbonate, base excess, sodium, potassium, and calcium concentrations. Normal metabolic status was maintained throughout the study by taking blood samples every 30-60 min and correcting any abnormal value.

The chest was opened through a median sternotomy, and the heart was suspended in a pericardial cradle. For delivering the defibrillation shocks, round mesh titanium patch electrodes, $4.5 \mathrm{~cm}^{2}$, were secured to the epicardium of the right atrium as the anode and to the ventricular apex as the cathode. Two pairs of pacing wires were inserted into the $\mathrm{RV}$ and left ventricular (LV) free walls for electrical stimulation to induce VF. A pair of sensing wires was inserted into the RV anterior wall so that shocks could be given during sinus rhythm at a predetermined interval after the last sensed depolarization to determine the impedance of the heart and defibrillation electrodes.

Truncated exponential defibrillation shocks of 5-ms duration were generated by a special device built by Intermedics, Inc. (Freeport, TX) To reduce amplifier saturation, most of the mapping system was disconnected during the shock (9). Blanking started $2 \mathrm{~ms}$ before the beginning of the shock and stopped $5 \mathrm{~ms}$ after the end of the shock. During this 12-ms period, the front-end filters were disconnected so that they would not be saturated by the shock. Because it took $\sim 15 \mathrm{~ms}$ for the signals to return to baseline, effective recording started $20 \mathrm{~ms}$ after reconnection of the amplifiers.

\section{Part I: epicardial activation and septal activation}

Six dogs were used in this part of the study. Two defibrillation patch electrodes were secured to the epicardium. One was sutured to the right atrium as anode and the other to the ventricular apex as cathode. This electrode configuration was used because it creates a relatively simple shock field that is strongest at the apex and weakest at the base of the ventricles. Before beginning the defibrillation protocol, shocks of 20 and
$100 \mathrm{~V}$ were given during sinus rhythm, $300 \mathrm{~ms}$ after the last sensed depolarization. These shocks were used to set the amplifier gains and to determine the impedance. This impedance value was used to predict the voltage needed to deliver shocks of desired energy levels. VF was induced by programmed pacing with 10 successive stimuli $250 \mathrm{~ms}$ apart followed by an extrastimulus in the vulnerable period (16). The defibrillation shock was given 10-15 s after the onset of VF. The voltage and current of the shock were recorded on the oscilloscope, and the actual energy delivered was calculated as their product multiplied by time $(0.005 \mathrm{~s})$. If the shock was unsuccessful, defibrillation was achieved within $30 \mathrm{~s}$ with a shock of 20-30 J delivered through a pair of hand-held paddle electrodes on the pericardium. Based upon the findings that ventricular excitability and vulnerability are not altered immediately after fibrillation and defibrillation (17), and that a 2-min recovery period is sufficient to maintain a stable defibrillation threshold (13), we waited for at least $4 \mathrm{~min}$ between each fibrillation-defibrillation episode.

Defibrillation with sock and septal plunge electrode recordings. For the first two experiments, only the sock electrode array and septal plunge electrodes were used. The first shock was given with a predicted energy of $1 \mathrm{~J}$. Shock strength was then increased in increments of $1 \mathrm{~J}$ until defibrillation was achieved. The energy delivered by this shock was defined as the defibrillation threshold. The last unsuccessful shock was defined as the subthreshold shock. The shock strength was then increased in increments of $1 \mathrm{~J}$ until the delivered energy was twice the defibrillation threshold. A second set of shocks was given in a random sequence with a predicted strength ranging from $1 \mathrm{~J}$ to twice threshold in $1-\mathrm{J}$ increments. In one dog with a high defibrillation threshold, we omitted the second set of shocks to avoid an excessive number of defibrillation episodes for that dog. A third set of shocks was then given with energies from $1 \mathrm{~J}$ above the defibrillation threshold to three times the threshold energy in 1-J increments. Higher-energy shocks were performed last because they were the most likely to cause myocardial damage (18).

Defibrillation with sock, septal plunge, and plaque electrode recordings. For the next four experiments, the plaque electrode array was applied to the posterobasal aspect of the ventricles in two dogs and to the anterobasal aspect of the right ventricle in the other two dogs. These plaque sites were chosen because they were the regions in which epicardial activation first appeared after the shock in the first two experiments. The first shock was given with a predicted energy of $6 \mathrm{~J}$. Shock strength was then increased in 1-J increments until three episodes of successful defibrillation were achieved. If there were less than three episodes of unsuccessful defibrillation at this point, the shock strength was decreased $1 \mathrm{~J}$ at a time beginning at $5 \mathrm{~J}$ until a total of three unsuccessful defibrillation episodes were recorded. Upon completion of the defibrillation protocol for the plaque, the sock electrode array and plunge electrodes were then applied and the defibrillation protocol was performed as for the first two dogs.

Data analysis. The dog was killed, and the locations of the epicardial recording electrodes were marked. The heart was'then excised and weighed. The plunge electrodes were removed, but the anchors were left in place. For the one plunge without an anchor, the epicardial entrance was marked. After recording the electrode locations on a diagram of the ventricles, we dissected the heart to expose both sides of the interventricular septum. The locations of the anchors and the epicardial entrances of the plunge electrodes were recorded.

For recordings from sock and plunge electrodes, the recordings from each channel were displayed on a computer terminal (model 4014, Tektronix, Inc., Beaverton, $O R$ ) and the seven activations immediately before and after the shock were identified $(8,19)$ for the first and the last episode of unsuccessful defibrillation. The average cycle length of VF before the shock was compared for these two episodes. The average cycle length of the first VF episode was designated the baseline VF cycle length (Fig. 1). For the other episodes, the activation before and the first five activations immediately after the shock were identified. All activations after the shock were timed with reference to reconnection of the amplifiers as time 0 .

The effect of the shock on the rate of epicardial activation was demonstrated by comparing the intervals between activations ( $R R$ intervals) 


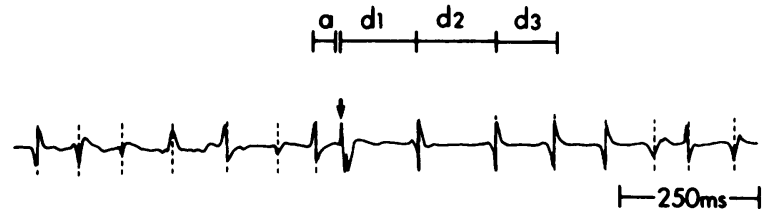

Figure 1. Definition of preshock and postshock intervals. Reconnection of the amplifiers $5 \mathrm{~ms}$ after the end of the shock is indicated by the arrow. Seven activations before and after the shock are shown. The activation time selected for each complex is shown by a dashed line. The baseline RR interval (baseline VF cycle length) was calculated by averaging the six intervals of all channels before the shock. The interval between the last preshock activation and the shock is labeled $a$, between reconnection of the amplifiers $5 \mathrm{~ms}$ after the shock and the first postshock activation is labeled $d l$, between the first and second postshock activations is labeled $d 2$, and between the second and third postshock activations is labeled $d 3$. If $d 2$ or $d 3$ was longer than the baseline RR interval plus two SD, it was said to be significantly delayed. The minimum value of $d l$ for all channels was called the isoelectric window (ww) after the shock. The artifact after the shock was caused by reconnection of the amplifier.

after the shock to the baseline VF cycle length (Fig. 1). The shortest first postshock interval was identified by examining all of the channel recordings and was called the isoelectric window width (ww) for that shock (Fig. 1). Isochronal maps were drawn for all five activations after successful shocks, and for the first two activations after unsuccessful shocks (20). The early activation sites, defined as sites that activated earlier than the surrounding electrode sites, were determined for each isochronal map. Because each early site gave rise to an activation front, the number of activation fronts equaled the number of early sites until the number of activation fronts changed because of collisions or block. The time required to activate both the septum and the epicardium (the cardiac activation time) was determined by subtracting the time of earliest recorded activation of either the septum or epicardium from the latest recorded activation time for each map. The areas directly depolarized by the $20-\mathrm{V}$ and $100-\mathrm{V}$ shocks delivered during sinus rhythm were also calculated to indicate regions of high- and low-field strength generated by the shock (9).

For recordings from plaque electrodes, two activations were analyzed immediately before and five immediately after the shock from three successful and three unsuccessful defibrillation episodes for each dog. Atrial recordings could be obtained in one dog because the plaque was placed partially across the atrioventricular sulcus. An additional $11 \mathrm{ep}-$ isodes of defibrillation were analyzed for this dog to evaluate atrial activity during the immediate postshock period.

Student's $t$ test (21), analysis of variance (ANOVA) using the repeated measure design, general linear model procedure, and Wilcoxon sign rank test (22) were used to analyze the differences of the means.

\section{Part II: transmural activation}

Five dogs were used in part II. The same defibrillation electrode locations were used as for part I. A sock containing 24 bipolar button electrodes was placed on the RV outflow tract and LV anterobasal wall. These sites were selected because in part I they were frequently the location of earliest activation after the shocks. To shorten the length of the study, VF was induced by 60 -cycle alternating current. Subthreshold defibrillation shocks were given 10-15 s after the onset of VF, and epicardial isochronal maps were generated during the study. We determined which electrode repeatedly recorded the earliest activation after the shock. This electrode was called the index electrode. Four plunges were inserted around the index electrode and eight bipolar recordings were made from each plunge. 18-34 shocks of the same strength (range 2-5 J) were then given. Isochronal maps were generated during the study to confirm that adequate plunge and sock electrode recordings were being made around the index electrode.
Data analysis. Upon completion of the defibrillation protocol, an incision was made into the RV outflow tract along the atrioventricular sulcus. The endocardium of the RV free wall was examined to determine the exit point of each plunge electrode. The epicardial entrances of the plunge electrodes and the locations of the sock recording electrodes were then marked. Seven activations were identified during VF in one fibrillation-defibrillation episode, and the cycle length was calculated. For all other episodes, the activations immediately before and after the shock were identified, and isochronal maps were drawn for the first postshock activation. For each episode we determined whether an early epicardial site of activation after the shock was at the index electrode, at an electrode adjacent to the index electrode, or at an electrode away from the index electrode. Those episodes with early sites away from the index electrode were excluded from further analysis. To determine endocardial and intramural activation around the early epicardial site, the recordings of plunge electrodes adjacent to the early epicardial sites were analyzed.

\section{Results}

\section{Part I: epicardial and septal recordings}

A total of 104 unsuccessful and 116 successful defibrillation episodes were analyzed with sock electrode recordings. Out of 12,320 channels of epicardial and 1,760 of septal recordings, $10,198(83 \%)$ and $840(48 \%)$, respectively, were adequate for analysis. Inadequate recordings included missing signals, signals too small for analysis, and broken wires. Missing signals could be caused by poor contact of the electrode with the tissue, or the absence of myocardium adjacent to the electrode when electrodes were over the atrioventricular sulcus (Fig. 2).

EFFECT OF SHOCKS ON THE RATE AND SYNCHRONY OF EPICARDIAL ACTIVATION. The cycle length (the interval between successive activations in the same channel) of the first VF episodes averaged $105 \pm 16 \mathrm{~ms}$, not significantly different from $106 \pm 12 \mathrm{~ms}$ of the last VF episodes of each $\operatorname{dog}(P=0.74$ by paired $t$ test). In contrast to humans (23), the VF cycle length at the base (105 $\pm 14 \mathrm{~ms})$ was not significantly different from that at the apex ( $105 \pm 17 \mathrm{~ms}, P=0.87$ by paired $t$ test). Both successful and unsuccessful shocks delayed epicardial activation between the first two postshock activations ( $d 2$ in Fig. 1). For unsuccessful defibrillation episodes, the percentage of channels that recorded a prolonged immediate postshock interval increased as the shock strength increased (Fig. 3).

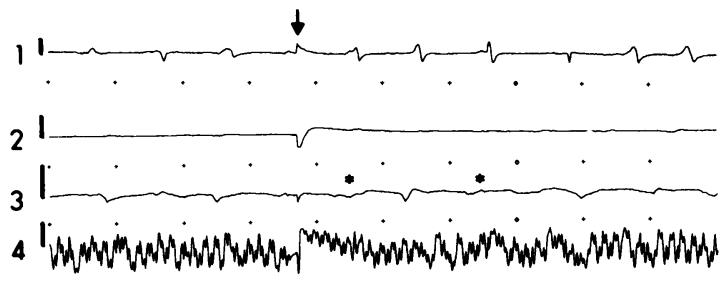

Figure 2. Examples of inadequate sock electrode recordings. The time of reconnection of the amplifiers after the shock is shown by an arrow. Channel 1 is a good recording obtained by an electrode adjacent to that of channels 3 and 4 . Channel 2 is an inadequate recording from an electrode located at the base of LV, overlying the fat in the atrioventricular sulcus. Channel 3 shows signals too small to analyze (marked by an asterisk), so that the presence or absence of local activation could not be determined: Channel 4 is an example of 60 -cycle noise recorded by an electrode with a broken wire. The calibration marks in front of each channel are $10 \mathrm{mV}$ in amplitude. The dots between the channels are spaced $100 \mathrm{~ms}$ apart. 


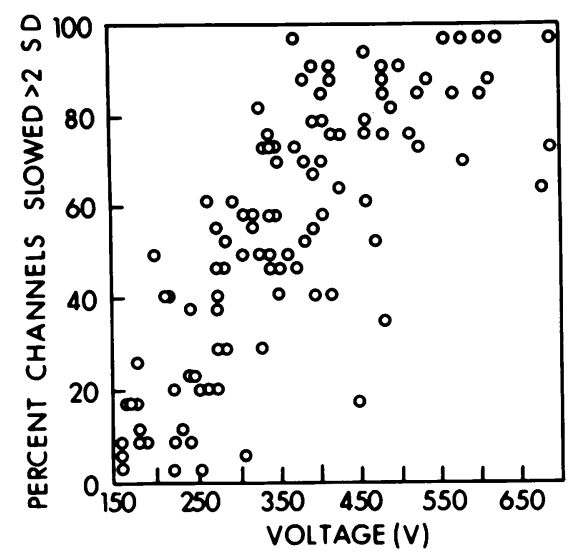

Figure 3. The effect of shock strength on the rate of epicardial activation for unsuccessful defibrillation episodes. On the ordinate is the percentage of recording channels that recorded a $d 2$ interval $2 \mathrm{SD}$ longer than the baseline VF cycle length. On the abscissa is the shock voltage delivered for that episode. There is a significant correlation between these two with an $r$ value of $0.77(P<0.0001)$.

PRESENCE OF A POSTSHOCK ISOELECTRIC WINDOW. Sock recordings. We found that immediately after reconnection of the amplifiers after the shock, an interval occurred during which no epicardial activation was recorded by any sock electrode (Fig. 4). The isoelectric window (ww) was present after all shocks, both successful as well as unsuccessful. The mean ww for all shocks was $209 \pm 253 \mathrm{~ms}$. This window is much larger than the mean interval between successive activation times during VF before the shock if the activation times for all 60 electrodes are combined and listed sequentially $(1.7 \pm 3.2 \mathrm{~ms})$.

The mean ww after unsuccessful $(64 \pm 22 \mathrm{~ms})$ and successful $(339 \pm 292 \mathrm{~ms})$ shocks were significantly different $(P<0.02)$ by the Wilcoxon sign rank test (22). The longest ww observed after an unsuccessful shock was 130 ms. In 81 of the 116 successful defibrillations, reconnection of the amplifiers was followed by an isoelectric ww of $>130 \mathrm{~ms}$. The other 35 successful episodes were characterized by a ww of $<130 \mathrm{~ms}$ followed by one to three rapid activations. We call the former recovery type A (Fig. $4 \mathrm{~A}$ ) and the latter type B (Fig. $4 \mathrm{~B}$ ). After the isoelectric window of type $A$ and the rapid postshock activations of type B, supraventricular or idioventricular activations with a relatively regular $\mathrm{RR}$ interval appeared (last seven activations of Fig. $4 \mathrm{~A}$ and last five activations of Fig. $4 \mathrm{~B}$ ). Activations synchronized transiently after all unsuccessful shocks, but soon returned to the less organized preshock distribution (Fig. $4 C$ and $D$ ).

Isochronal maps of the first depolarization after an unsuccessful shock indicated that activation first appeared on the epicardium at one to five sites (mean of $2.1 \pm 0.9$ sites) and spread centrifugally away from these early sites as large, coherent activation fronts. For most of the 104 mapped episodes of unsuccessful defibrillation, the earliest site of activation was recorded near the atrioventricular sulcus, primarily over the RV outflow tract or the LV posterior wall (Fig. 5). For 79 of the 116 episodes of successful defibrillation, the early sites after the isoelectric window were also at the base of the ventricles (Fig. $6 \mathrm{~A}$ ). The early sites for the other 37 episodes of successful defibrillation were in the lower part of the ventricles (Fig. $6 \mathrm{~B}$ ). A major factor associated with the postshock epicardial activation pattern was the ww (Fig. 7). For all shocks, both successful and unsuccessful,

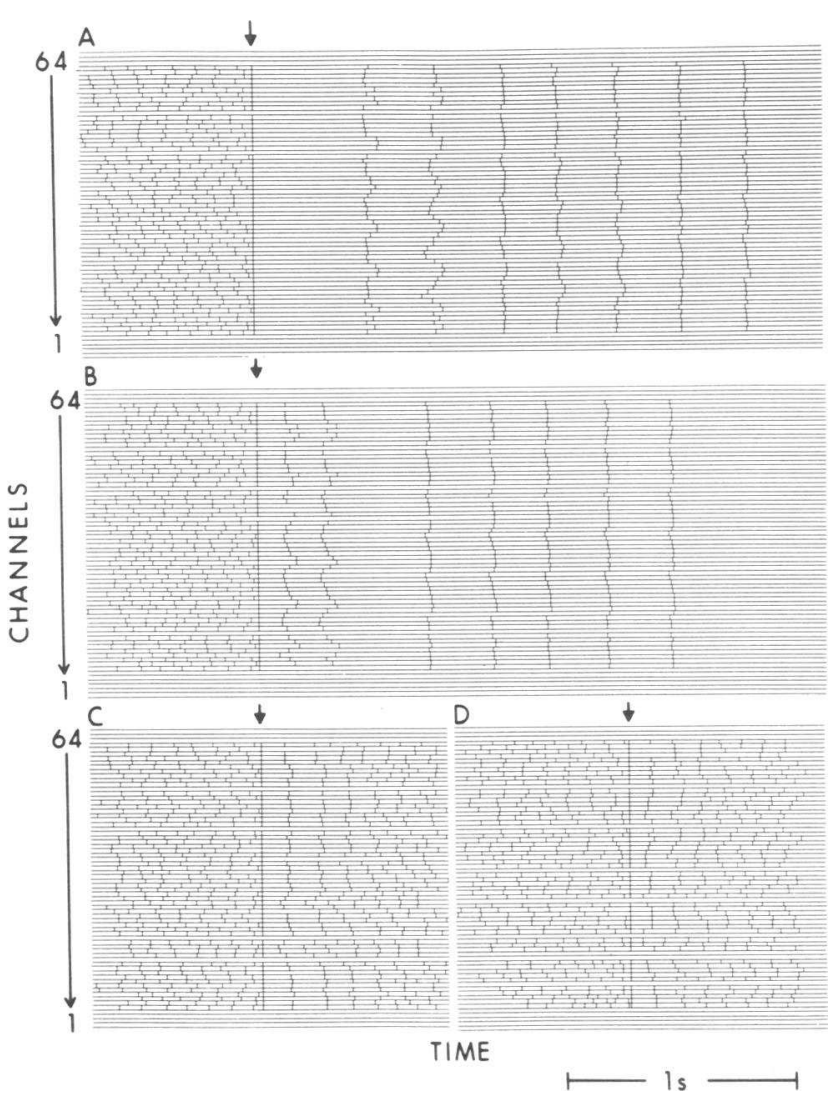

Figure 4. Effect of shocks on epicardial activation times. The horizontal lines separate the recording channels. The vertical hash marks are the times of individual activations recorded from each channel. The time of amplifier reconnection after the shock is shown by the vertical line beneath the arrow. Seven activations are shown before and after the shock. ( $A$ and $B$ ) Examples of successful defibrillation episodes: $A$ shows a type $A$ recovery because the isoelectric window after the shock (18.2 J) is $>130 \mathrm{~ms} ; B$ shows a type B recovery with two rapid activations beginning $<130 \mathrm{~ms}$ after the shock (11 J). Examples of unsuccessful shocks are shown in $C$ (subthreshold shock, 7.3 $\mathrm{J}$ ) and $D$ (low energy shock, $1.3 \mathrm{~J}$ ). ( $C$ and $D$ ) Temporal clustering of the first activation after the shock with return to the preshock distribution by the third or fourth postshock activation.

$75 \%$ of the episodes with early sites at the ventricular bases had a ww $\leq 130 \mathrm{~ms}$ whereas $90 \%$ of the maps with early sites away from the bases had a ww $>130 \mathrm{~ms}$.

Plaque recordings. We analyzed 12 successful and 12 unsuccessful defibrillation episodes with plaque electrode recordings. Of the 1,344 plaque electrode recordings, $1,156(86 \%)$ were adequate for analysis. The major reason for inadequate recordings was placement of the plaque over the atrioventricular sulcus (Fig. 8). An isoelectric window was present after the shock in all cases. The ww varied from 49 to $117 \mathrm{~ms}$ for unsuccessful defibrillations and from 73 to $596 \mathrm{~ms}$ for successful defibrillations. As was found with the sock electrode recordings, all shocks that produced a ww longer than 130 ms successfully defibrillated. Discrete early sites of activation were present after the isoelectric window with coherent, synchronized activation fronts spreading away from these sites (Fig. 9).

Thus, mapping with the more closely spaced electrodes of the plaque confirmed the presence of an isoelectric window on the epicardium after the shock and furnished no evidence for 
A
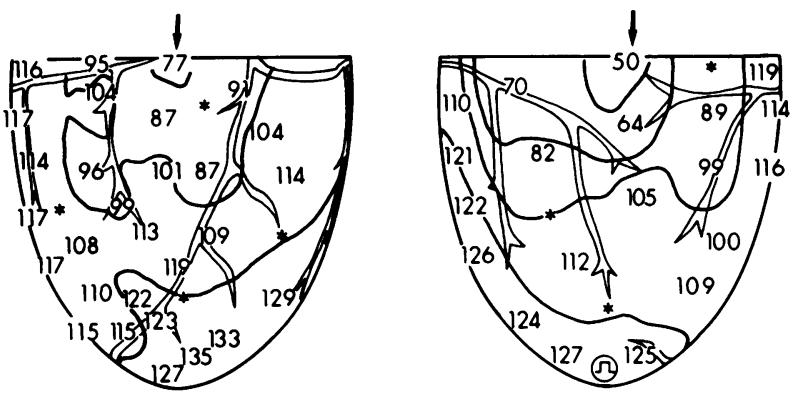

B

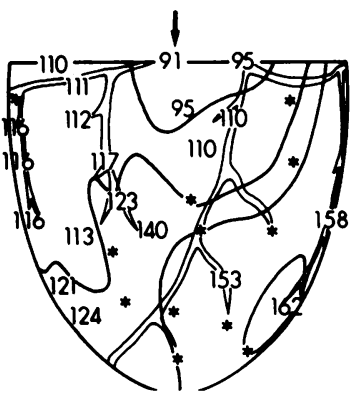

C

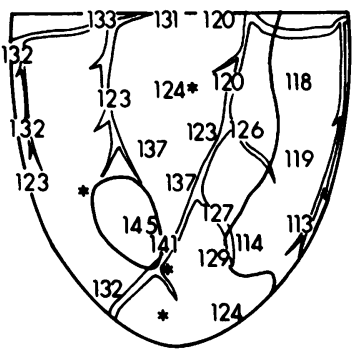

A

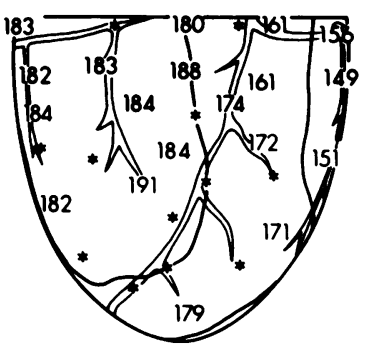

B
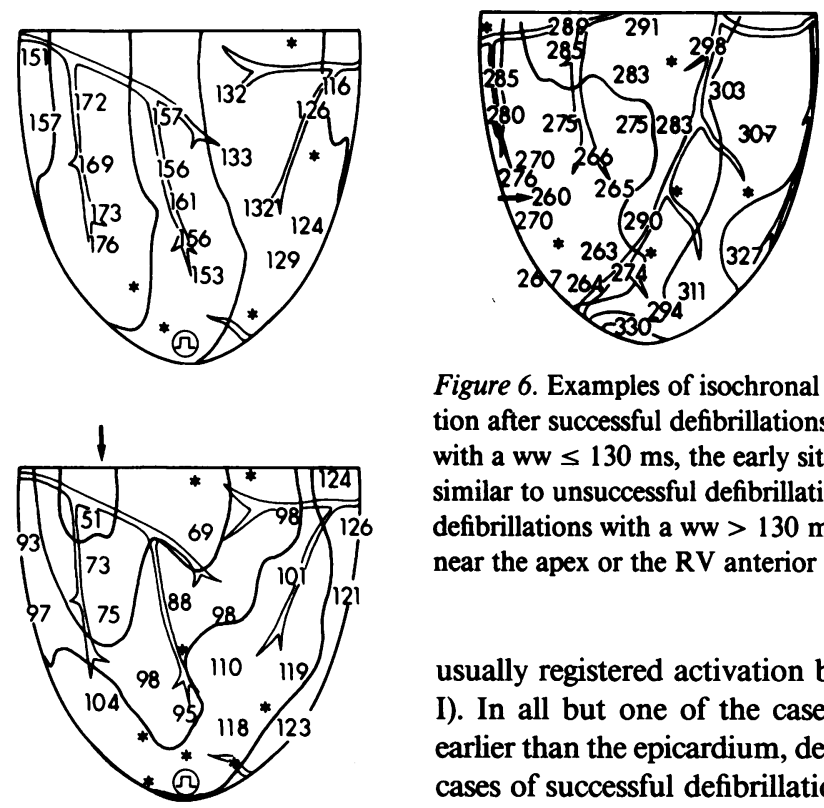

Figure 5. Examples of postshock isochronal maps after unsuccessful defibrillation shocks. The maps are displayed as two complementary projections of the heart with the anterior LV and RV epicardium shown in the left diagram and the posterior $L V$ and $R V$ epicardium in the right diagram. Locations of the apical defibrillation electrode is indicated by a square wave symbol within a circle. Numbers represent the locations of electrodes with satisfactory recordings and give the time of activation for those locations timed from amplifier reconnection after the shock. Arrows indicate the early activation sites, and the activation time of the earliest of these sites is the ww for this episode. Asterisks indicate electrode sites where adequate recordings were not obtained. The isochronal lines are $20 \mathrm{~ms}$ apart. $(A)$ With a 4.6-J shock, there were two early sites; one at the RV outflow tract, and the other at the LV posterior wall near the crux. $(B)$ With a 17.6-J shock, only one early site was observed; it was at the RV outflow tract. $(C)$ With a 9.8-J shock, a single early site was noted at the LV posterior wall, near the atrioventricular sulcus. In all cases, activation spread away from the early sites in large, synchronized depolarization fronts.

small epicardial reentrant pathways during the isoelectric window that could have been missed by the more widely spaced sock electrode recordings. During the second or third activation after the unsuccessful defibrillation, however, block and possible reentry were sometimes observed.

Septal plunge recordings. During normal sinus rhythm in three of the six dogs, activation was recorded earlier at a septal electrode than at any of the epicardial electrodes. For the first postshock activation, however, an electrode on the epicardium
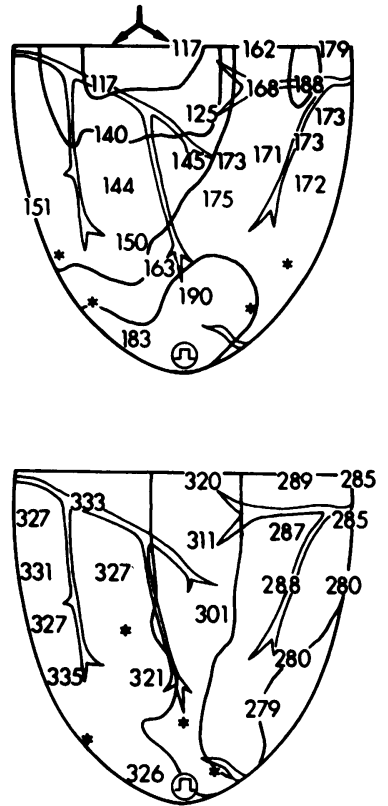

Figure 6. Examples of isochronal maps of the first postshock activation after successful defibrillations. $(A)$ For successful defibrillations with a ww $\leq 130 \mathrm{~ms}$, the early sites were usually located at the base, similar to unsuccessful defibrillations (11.7-J shock). (B) For successful defibrillations with a ww $>130 \mathrm{~ms}$, the early sites were usually located near the apex or the RV anterior wall (18.2-J shock).

usually registered activation before any septal electrode (Table I). In all but one of the cases in which the septum activated earlier than the epicardium, defibrillation was successful. In those cases of successful defibrillation in which the septum activated first, the ww was $>130 \mathrm{~ms}$. For unsuccessful ventricular defibrillation and for successful defibrillation with a ww $\leq 130 \mathrm{~ms}$, respectively, the earliest recorded activation in the septum was an average of $37 \pm 19 \mathrm{~ms}$ and $38 \pm 19 \mathrm{~ms}$ later than the earliest recorded activation on the epicardium. These times were significantly different $(P<0.001$ for both comparisons) from that for successful defibrillation with a ww $>130 \mathrm{~ms}$, when the septum activated an average of $18 \pm 19 \mathrm{~ms}$ later than the epicardium. Thus, an isoelectric window was present in septal recordings, and the septum was usually not the site of earliest activation following the shock.

RELATIONSHIP OF WINDOW WIDTH AND SHOCK STRENGTH TO THE OUTCOME OF DEFIBRILLATION. The ww was related to the outcome of the defibrillation attempts. All episodes with a ww $>130 \mathrm{~ms}$ were successful. The isochronal maps for successful shocks with ww $>130$ ms were different from the maps for successful shocks with ww $\leq 130 \mathrm{~ms}$ (Fig. 6).

Besides ww, shock strength expressed as energy, voltage, or current was also related to the outcome of the defibrillation attempts; low shock strength was associated with unsuccessful defibrillation while high shock strength was associated with successful defibrillation (Fig. 10). The size of the region of overlap in which either success or failure of defibrillation could occur was smaller for ww than for shock strength. All episodes with a ww $<63 \mathrm{~ms}$ were unsuccessful whereas all episodes with a ww $>130 \mathrm{~ms}$ were successful. Only $38 \%$ of the episodes had a ww in the overlapping region of $63-130 \mathrm{~ms}$, which was associated with both successful and unsuccessful defibrillation. The region of overlap was greater for shock energy. All episodes with a shock 
A
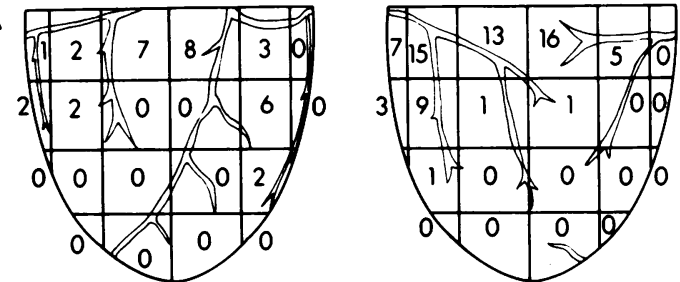

B

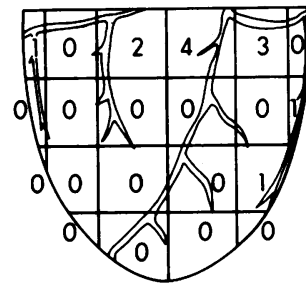

C

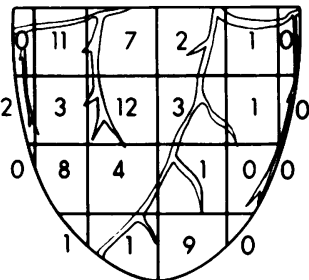

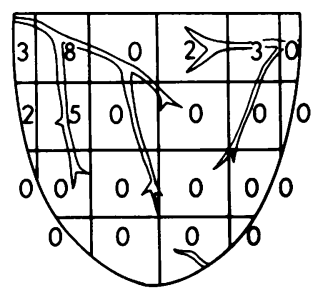

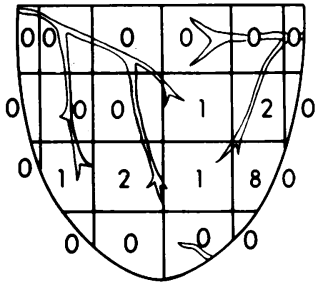

Figure 7. Location of the earliest sites for the first postshock activation. The epicardium is divided into regions by grid lines. The numbers specify the number of defibrillation episodes in which the site of earliest recorded postshock activation was located within the region for a particular group of shocks. $(A)$ Distribution of earliest sites after unsuccessful defibrillation shocks. The earliest sites were usually located in the basal half of the ventricles near the atrioventricular sulcus. Earliest sites were most frequent over the RV outflow tract anteriorly or near the crux posteriorly. $(B)$ Distribution of earliest sites after successful defibrillation shocks with a ww $\leq 130 \mathrm{~ms}$. Most of earliest sites were also at the base. $(C)$ Distribution of earliest sites after successful defibrillation shocks with a ww $>130$ ms. The earliest sites occurred in either the basal or the apical halves of the ventricles.

energy $<5.6 \mathrm{~J}$ were unsuccessful and all those $>21.3 \mathrm{~J}$ were successful; a total of $64 \%$ of the episodes were in the region of overlap of 5.6-21.3 J. A wide region of overlap was also present

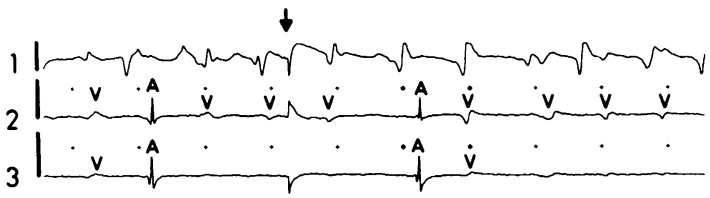

Figure 8. Examples of inadequate ventricular recordings owing to placement of the plaque over the atrioventricular sulcus. Channel 1 shows an adequate ventricular recording. Channel 2 overlies the atrioventricular sulcus and records both atrial $(A)$ and ventricular $(V)$ activations. Channel 3 recorded good atrial but poor ventricular activations. The latter two channels are inadequate for analyzing activation during defibrillation because an atrial complex may obscure a ventricular activation. The calibration marks in front of each channel are 10 $\mathrm{mV}$ in amplitude. The time of amplifier reconnection after the shock is shown by the arrow.
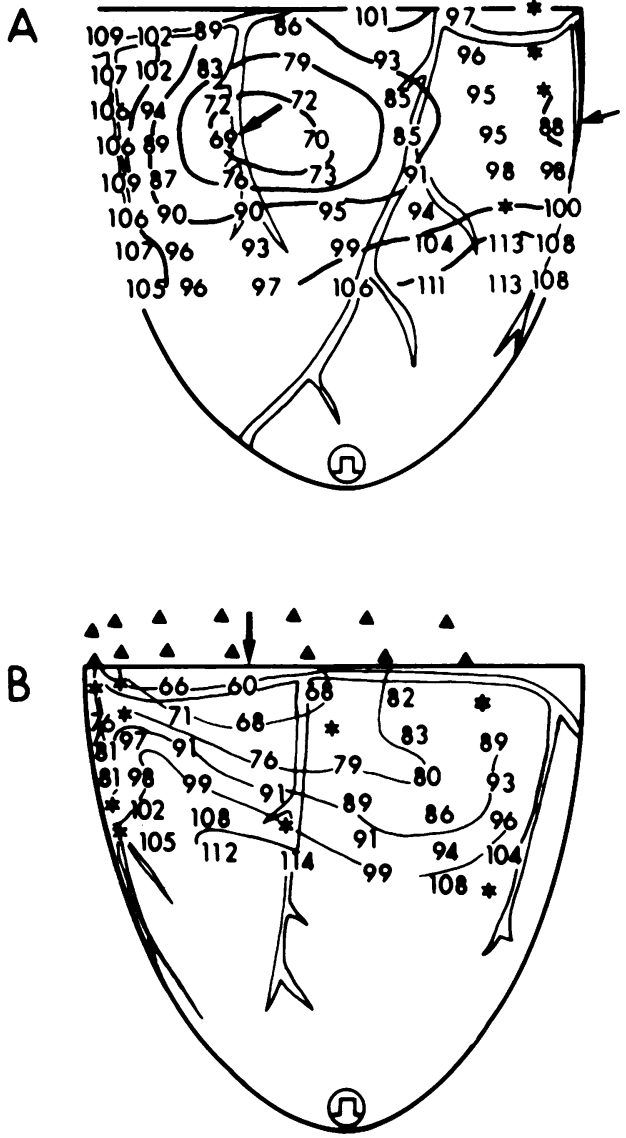

Figure 9. Postshock isochronal maps of unsuccessful defibrillation with plaque electrode recordings. Isochronal lines are $10 \mathrm{~ms}$ apart. $(A)$ An early activation site (large arrow) at the RV outflow tract after a shock of $7 \mathrm{~J}$. At the edge of the plaque another early site (small arrow) probably arose from an activation front that started from the LV posterior wall in a region not recorded by this plaque. $(B)$ An early site (arrow) at the margin of the atrioventricular sulcus recorded during another unsuccessful defibrillation episode. The upper two rows of electrodes (triangles) did not record ventricular electrical activity because they were placed across the atrioventricular sulcus.

for shock voltage and current (Fig. 10). The region of overlap for ww, besides being smaller than for shock strength, was also more constant from dog to dog. Thus ww was a better indicator of the outcome of a defibrillation attempt than was the strength of the shock.

CORRELATION BETWEEN THE WINDOW WIDTH AND ENERGY. In the 81 successful type A defibrillation episodes,

Table I. Location of Earliest Postshock Activation

$\begin{array}{lr}\begin{array}{l}\text { Septum } \\ \text { activated first }\end{array} & \begin{array}{l}\text { Epica } \\ \text { activa }\end{array} \\ & \\ 12 & 69 \\ 0 & 35 \\ 1 & 103\end{array}$

* $P<0.001$ compared with unsuccessful and type B defibrillation by the Chi square test. 


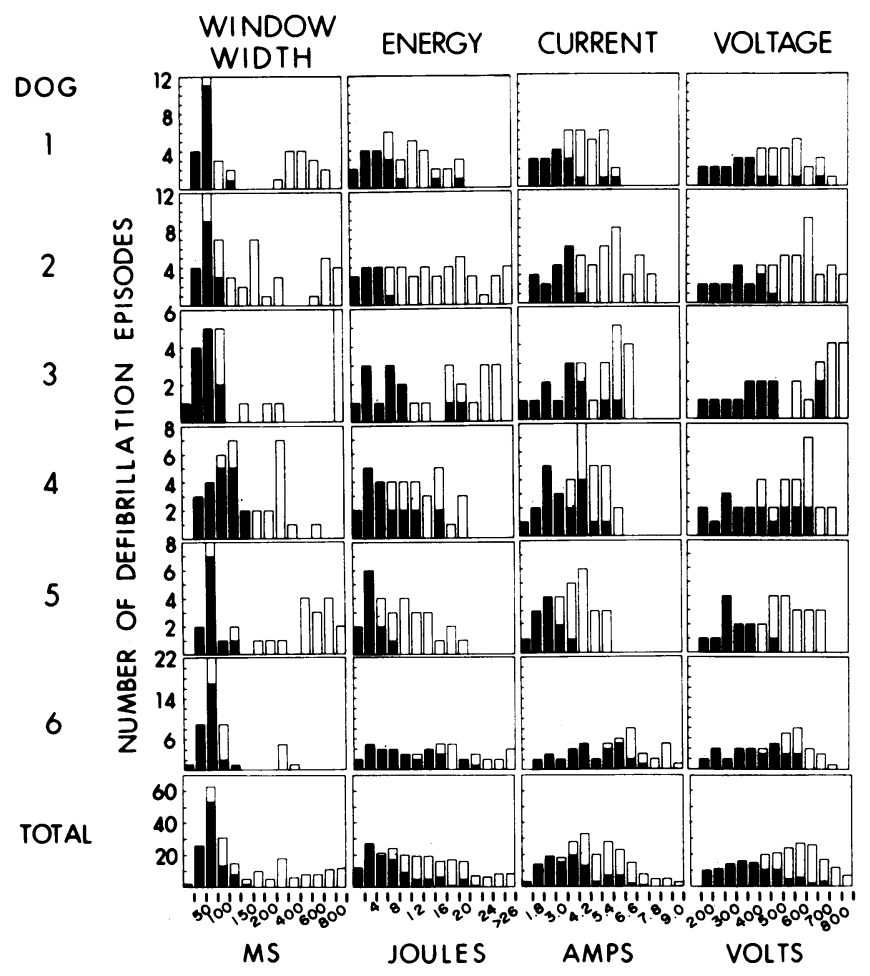

Figure 10. Window width and shock energy, voltage, and current for successful and unsuccessful defibrillation. The ordinate gives the number of shock episodes for each dog with window width, energy, current, and voltage in the range shown on the abscissa. The number of shocks that were unsuccessful in halting fibrillation is shown in black, and the number of successful shocks is shown in white. The bottom row gives the total results for all dogs. the energy was $16.7 \pm 5.9 \mathrm{~J}$, which is significantly higher $(P$ $<0.025$ with ANOVA) than the $14.2 \pm 6.1 \mathrm{~J}$ associated with the 35 episodes with successful type B defibrillation. For unsuccessful defibrillation, but not for successful defibrillation, there was a significant correlation between the ww and the energy, with the correlation coefficients ranging from 0.53 to 0.95 for each of the six dogs (Fig. 11). When the results for all dogs were pooled, the correlation coefficient was only 0.52 because the slopes and intercepts of the regression lines differed markedly among the dogs. The pooled correlation coefficient for ww and voltage was 0.65 , and for ww and current was 0.43 .

DIFFERENCES BETWEEN UNSUCCESSFUL AND SUCCESSFUL DEFIBRILLATION EPISODES. Although the locations of the sites of early activation after the isoelectric window that followed type B successful shocks were different from those following type A successful shocks, they were not different from those that followed unsuccessful shocks (Fig. 7). Successful type B defibrillation episodes were different from unsuccessful episodes in several other ways, however (Table II). For the successful defibrillation episodes, the mean energy delivered was higher; the ww for the first two postshock activations was longer; the mean number of early sites for the first two postshock activations was smaller; and the mean time for the first postshock activation to depolarize the entire epicardium and septum, i.e., the cardiac activation time, was longer.

For unsuccessful defibrillation, the number of early sites for the first postshock activation decreased as energy increased ( $r$ $=-0.59, P<0.001)$. The early sites for the first and second postshock activations after unsuccessful defibrillation shocks often occurred at the same or at adjacent electrodes (Table III). This was not true for successful defibrillation. By multiple regression analysis (24), cardiac activation times for the first and
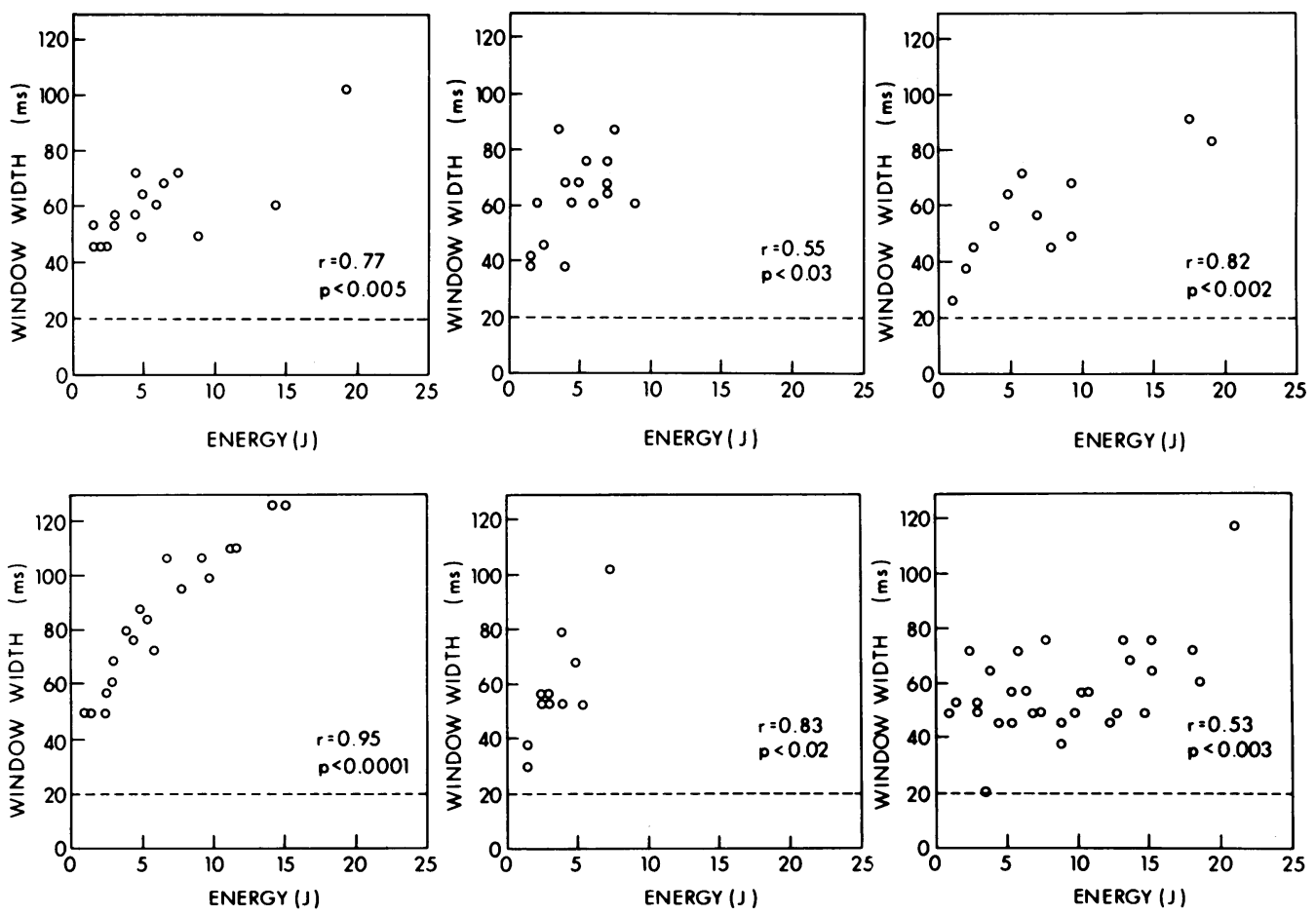

Figure 11. The relationship between ww (ordinate) and shock energy for unsuccessful shocks (abscissa). Each panel represents a different dog. The horizontal dashed line indicates the end of the $20 \mathrm{~ms}$ interval

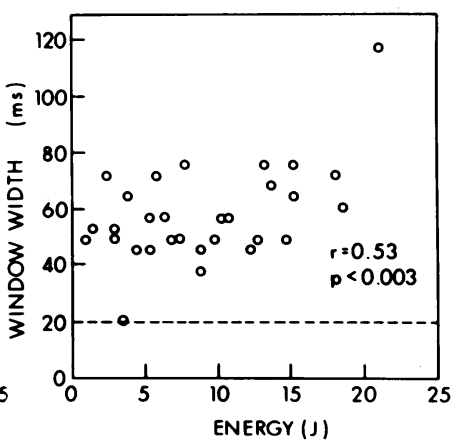

after the shock in which activations could not be detected reliably by the mapping system. 
Table II. Comparison of Unsuccessful and Successful Type B Defibrillation

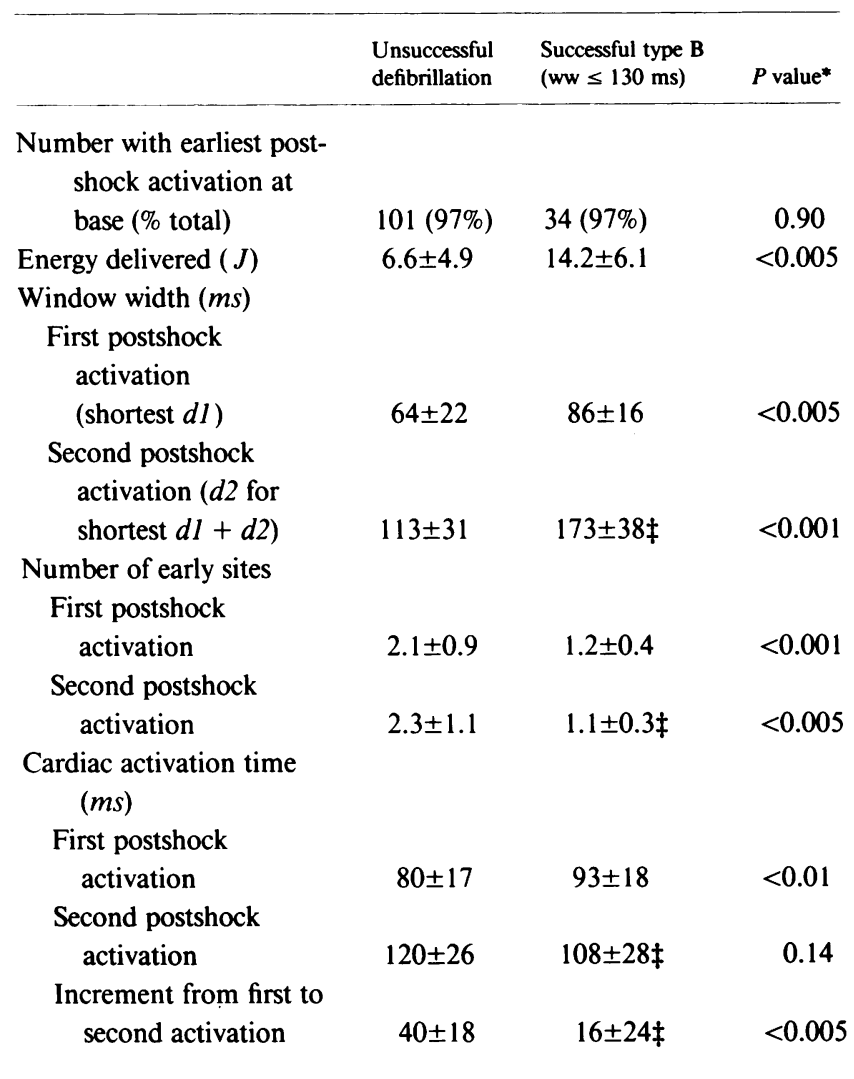

* By Student's $t$ test.

$¥ 12$ episodes had more than 1 postshock rapid activation to allow this analysis.

second postshock activations of the unsuccessful defibrillations were negatively correlated with both the number of early sites and the ww $(P<0.0001)$. Similar negative correlations were present for successful type B defibrillation, but were not statistically significant $(P=0.15)$ because of the smaller sample size. No such correlation was found for type A successful defibrillations.

COMPARISON OF SHOCK FIELD STRENGTH AND EARLY SITE LOCATIONS. In five of the first six experiments, adequate recordings were made when 20 - and $100-\mathrm{V}$ shocks were given during the TQ interval of the sinus rhythm. Because

Table III. Early Sites of the First and Second Postshock Activations

\begin{tabular}{llc}
\hline & $\begin{array}{l}\text { At same or } \\
\text { adjacent electrodes }\end{array}$ & $\begin{array}{l}\text { At different } \\
\text { electrodes }\end{array}$ \\
\hline $\begin{array}{l}\text { Unsuccessful episodes* } \\
\text { Successful episodes }\end{array}$ & 96 & 8 \\
$\quad \begin{array}{l}\text { Type A } \\
\text { Type B (1 rapid postshock } \\
\quad \text { activation) }\end{array}$ & 46 & 70 \\
$\begin{array}{l}\text { Type B ( }>1 \text { rapid postshock } \\
\text { activation) }\end{array}$ & 31 & 50 \\
\hline
\end{tabular}

* $P<0.005$ compared with the successful episodes by the Chi square test. the impedance of each heart varied, and because the defibrillation device did not deliver the exact voltage as programmed, the actual energy delivered by a $20-\mathrm{V}$ shock varied from 0.004 to $0.009 \mathrm{~J}$ (mean of $0.007 \mathrm{~J}$ ), and that by a $100-\mathrm{V}$ shock varied from 0.25 to $0.55 \mathrm{~J}$ (mean of $0.32 \mathrm{~J}$ ). The mean percentage of the total epicardial area directly depolarized by a $20-\mathrm{V}$ shock was $48.0 \pm 8.9 \%$ (Fig. 12), and by a 100 -V shock was $100 \%$. There was no significant correlation between the defibrillation threshold and the area directly depolarized by the $20-\mathrm{V}$ shock, even after normalization of the directly depolarized area by the actual delivered energy.

Because the shock field in the area directly depolarized by the shock is assumed to be stronger than the shock field in areas not directly depolarized (9), we studied the relationship between the early sites after defibrillation shocks and the area directly depolarized by the $20-\mathrm{V}$ shock during sinus rhythm. For unsuccessful defibrillation or successful type B defibrillation, the early sites were usually located in regions distant from the defibrillation electrodes, at the RV outflow tract or the basal part of the LV posterior wall. Both of these regions were outside the area directly depolarized by a $20-\mathrm{V}$ shock in sinus rhythm (Figs. 7 and 12). These results suggest that the early sites arose in those regions of the ventricles in which the shock field was weakest.

ATRIAL ACTIVATIONS DURING VENTRICULAR DEFIBRILLATION. The atrial response to the shock was observed in one dog in which a portion of the plaque was over the posterior left atrium. A total of 5 successful and 12 unsuccessful defibrillation episodes were analyzed. Among the five successful episodes, three were type A and two were type B. In all cases, the first occurrence of postshock ventricular activation preceded the first atrial activation (Fig. 8), suggesting that the first postshock ventricular activation was not conducted from the atria.

\section{Part II: transmural recordings}

The repeatability in location of the early sites with the defibrillation electrode configuration of part I allowed us to use plunge electrodes to record activation transmurally in part II.

PRESENCE OF A POSTSHOCK ISOELECTRIC WINDOW. In part II, 118 episodes of unsuccessful ventricular defibrillation were mapped. Of these, 49 were excluded from further analysis because none of the early sites of the first postshock epicardial activation was at or near the index electrode. Among the other 69 episodes, 32 had early sites at the index electrode and 37 had early sites at the electrodes adjacent to the index electrode. Out of 1,656 channels of epicardial and 2,208 channels of plunge

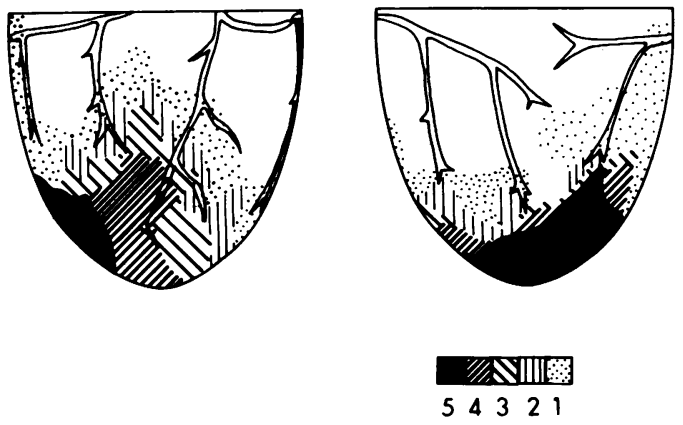

Figure 12 . The area directly depolarized by a $20-\mathrm{V}$ shock delivered during the TQ interval of normal sinus rhythm in five dogs. Different types of crosshatching represent the number of the dogs that had each area directly depolarized by the shock. 
electrode recordings of these 69 episodes, $1,342(81 \%)$ and 1,514 (69\%), respectively, were adequate for analysis. The VF cycle length $(101 \pm 10 \mathrm{~ms})$ was not significantly different from that of part I $(P=0.85)$.

In the 32 episodes in which the earliest recorded epicardial activation was at the index electrode, the mean ww recorded by the surrounding plunge electrodes $(58 \pm 16 \mathrm{~ms})$ was not significantly different from that recorded by the index electrode ( $59 \pm 19$ $\mathrm{ms}$ ). There was no evidence for continuous intramural or subendocardial electrical activity during the epicardial isoelectric window (Figs. 13 and 14). Although activation during VF before the shock did not occur in discrete cycles, the first postshock activation after the isoelectric window was synchronized.

In a few cases (Fig. 14), we identified two activation fronts traveling in different directions along the plunge that recorded the earliest activation. This finding is similar to the recordings made at the vortex of the leading circle during microreentry (25), but we did not have enough local recordings to tell if a complete reentry loop was present.

In 37 of the 69 episodes, activation was recorded slightly earlier from the plunge electrodes than from the epicardial electrodes. The earliest recorded activation for different episodes was located at all levels throughout the transmural extent of the plunges. There was no predilection for subendocardial sites as there was during sinus rhythm, when earliest activation was recorded from the most endocardial electrode in four of the five dogs. The preshock intervals of the electrode registering the earliest postshock activation were not randomly distributed. Rather, they clustered around $50 \mathrm{~ms}$ (Fig. 15).

\section{Discussion}

\section{Cessation and regeneration of $V F$}

The mechanism of electrical defibrillation is not known. The most commonly accepted hypothesis is based upon studies indicating that a critical mass of myocardium is necessary for the maintenance of fibrillation (26). Extrapolating these results to electrical defibrillation, it is assumed that a shock causes defibrillation when the activation fronts within a critical mass of myocardium are extinguished by direct depolarization of nonrefractory tissue within the critical mass (6). All activation fronts within the critical mass are extinguished because immediately after the shock none of the cells adjacent to the activation fronts are capable of being depolarized. According to this hypothesis, defibrillation fails solely because nonrefractory tissue in a critical mass of myocardium is not directly depolarized by the field of the shock, so that activation fronts can continue to pass through the myocardium after the shock and sustain fibrillation (27).

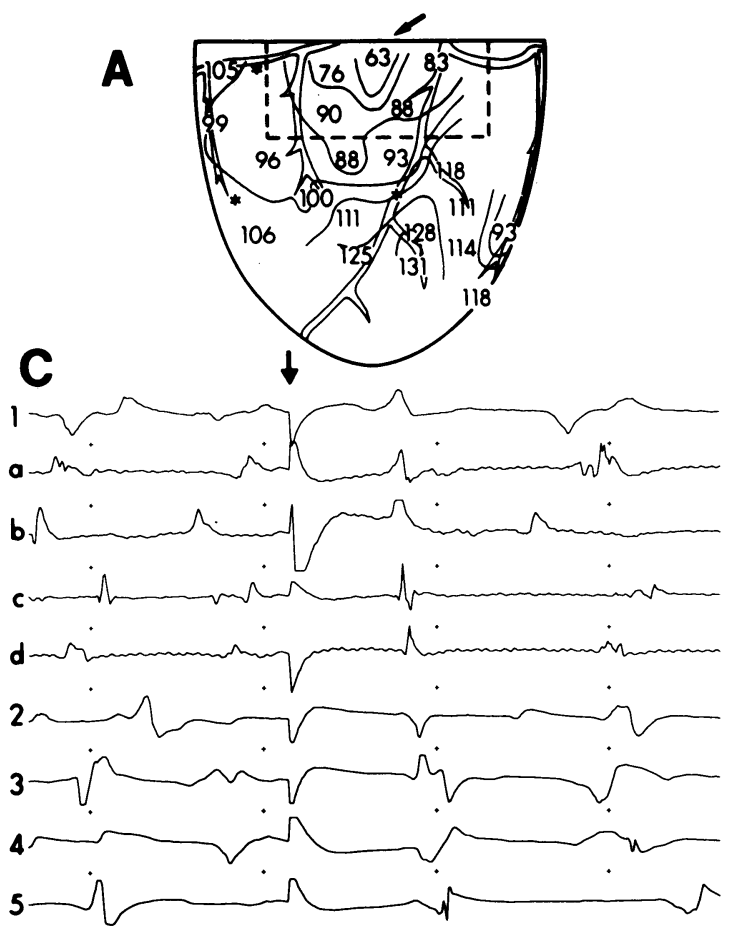

Figure 13. Transmural activation around an early epicardial site located at the upper part of $\mathrm{RV}$ outflow tract showing an isoelectric window in both epicardial and plunge electrode recordings. $(A)$ Epicardial isochronal map over the RV outflow tract and RV anterior wall for the first activation after the shock. An activation front arose near the top of the outflow tract (arrow). (B) Enlargement of the region within the dashed line in $A$ showing the relationship of the plunge electrodes to the epicardial electrodes in the region of earliest activation after the shock. The letters $a, b, c$, and $d$ mark the location of plunge electrodes, and the numbers 1-5 mark the location of epicardial button electrodes. The channel that recorded the earliest activation after the shock for each plunge electrode is displayed in $C$ along with the five
B
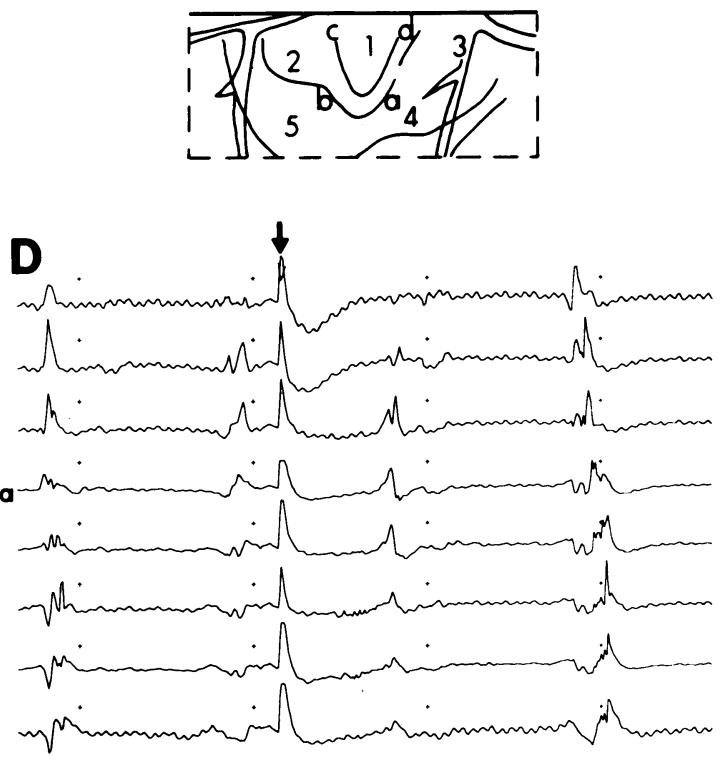

epicardial recordings. The arrow indicates the time of amplifier reconnection after the shock. The characters to the left of $C$ mark the location and type of the electrode, corresponding to $B$. Although the preshock activations of these nine channels spread over a 73-ms period, the postshock activations were synchronized, occurring within 17 ms of each other. (D) An 8-channel transmural recording from plunge $a$, which recorded the earliest postshock activation of any of the plunges. The channels of the plunge are displayed with the most endocardial electrode at the top and the most epicardial electrode at the bottom. The earliest activation was recorded intramyocardially, as indicated by the character $a$. 


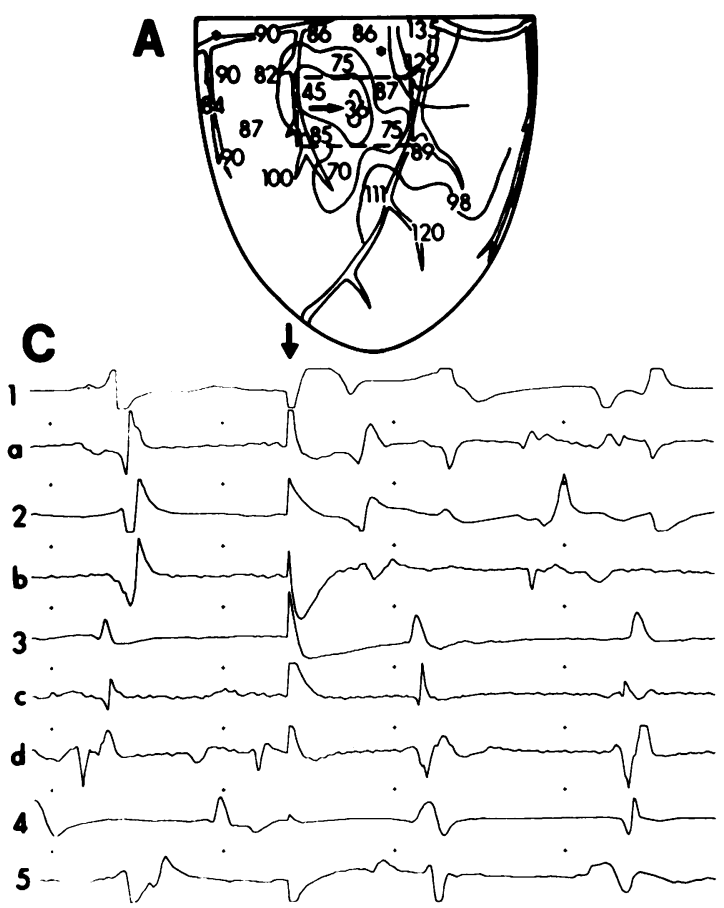

Figure 14. Transmural activation around an early epicardial site located at the lower part of the RV outflow tract. The panels are similar to those in Fig. 13. (D) Two activation fronts recorded by the same

This hypothesis has never been tested by cardiac mapping. To do so, we modified our mapping system to minimize amplifier saturation by the shock and recorded from the epicardium, septum, and RV endocardium and intramyocardium in the postshock period. Our results do not confirm the hypothesis that defibrillation fails because it does not annihilate activation fronts within a critical mass of the myocardium; rather, VF was transiently halted, as indicated by the isoelectric window, and then regenerated.

After both successful and unsuccessful defibrillation, an isoelectric window was observed in the immediate postshock period during which no activations were recorded from the epicardium, interventricular septum, intramyocardium, or endocardium. The

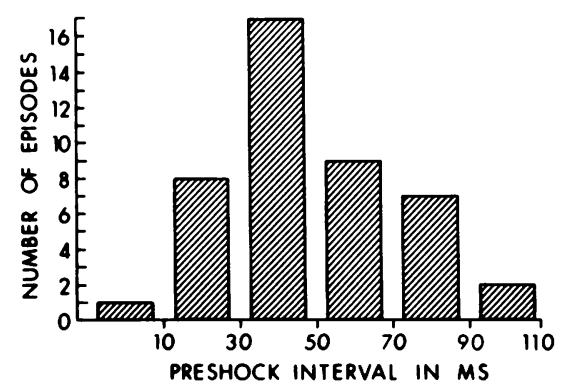

Figure 15. The interval between the last activation before the shock and the shock (labeled $a$ in Fig. 1) for the plunge or epicardial electrode recording the earliest activation after the shock. The number of defibrillation episodes is given to the left. These are the 32 unsuccessful defibrillation episodes in which earliest epicardial activation was recorded at the index electrode. If more than one electrode recorded the same early activation time, all such electrodes were included. The mean preshock interval was $50 \pm 24 \mathrm{~ms}$.
B
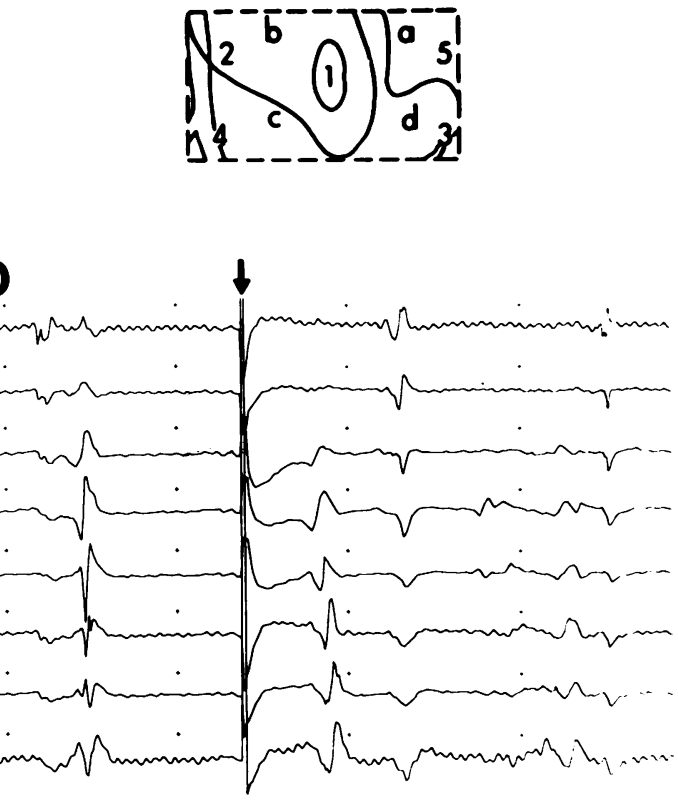

plunge in the immediate postshock period. The first front is earliest at the intramyocardium, as indicated by the character $a$; the second front is earliest at the endocardium.

isoelectric window averaged $64 \mathrm{~ms}$ after unsuccessful shocks. Windows of this length were never observed during VF before the shock; the interval between successive activations in different electrodes averaged $1.7 \pm 3.2 \mathrm{~ms}$. Although we cannot totally rule out the presence of a very slow activation front that was so small during the isoelectric window that it was not detected by our electrodes, the presence of the isoelectric window suggests complete cessation of all activation fronts in the immediate postshock period, even when the shock is unsuccessful. These findings do not support the critical mass hypothesis as the sole requirement for successful defibrillation. Although a sufficiently small shock presumably will not extinguish all activation fronts within a volume of myocardium, our results indicate that, for an epicardial defibrillation electrode combination, this energy is $<1 \mathrm{~J}$, much less than the energy required for defibrillation. While depolarization of a critical mass of myocardium may achieve defibrillation by chemical means as demonstrated by Zipes et al. (6), it is not the sole requirement for defibrillation by electrical means. In fact, the two examples of electrical shocks in the seminal paper by Zipes et al. (their Fig. 5) both show an isoelectric window, although it was not interpreted as such.

\section{Cause of the postshock isoelectric window}

There are at least two possible explanations for the isoelectric window. One is that some myocardial cells are responsive during the isoelectric window, but no stimulus is present to depolarize them. Because the RR intervals are so short during VF, much of the myocardium is probably refractory during the shock. According to this explanation, cells that were refractory at the time of the shock repolarize throughout the isoelectric window so that shortly after the shock some cells have recovered sufficiently to be activated by a stimulus. Because all activation fronts are extinguished by the shock, however, no stimulus is present to 
depolarize these cells until activation arises considerably later, thus producing an isoelectric window.

A second possible explanation is that the myocardium is unresponsive throughout the isoelectric window, having been electrically "paralyzed" by the shock. The response of the cell membrane to strong stimulation is different from its response to weaker stimulation. For example, after a 5-mA stimulus, van Dam et al. (28) observed no electrical activity during the next five to eight beats in the myocardium close to the stimulating electrodes. Lepeschkin et al. (2) observed similar electrical "standstill" after a defibrillation shock. Recently Moore and Spear (29) studied the effects of electric shock on the transmembrane potential. They found that after a 3.6-J stimulus given at phase 2, or a 2-J stimulus given at phase 3 of repolarization, the membrane potential "hung up" at its potential when the stimulus was given and recovery of the membrane potential to the true resting potential was delayed. Furthermore, until recovery was complete, it was not possible to evoke further all-or-none Purkinje fiber action potentials. These studies show that a defibrillation shock can have a long lasting effect on the cell membrane, even when it is delivered during phase 2 or 3 repolarization. This is quite different from the all-or-none response of the cell membrane to a low energy electrical stimulus. A defibrillation shock could thus produce membrane effects in all parts of the myocardium, even in those cells that are refractory at the time of the shock, so that a period of electrical silence, i.e., the isoelectric window, is produced.

\section{Mechanism for the postshock regeneration of $V F$}

The isoelectric window indicates that ventricular activation fronts are not present for an interval after the shock. Atrial recordings adjacent to an early ventricular site at the atrioventricular sulcus indicate that the first postshock activation did not come from the atrium via an accessory pathway. Thus the cells at the early ventricular sites must themselves be the source for the first postshock activation. After unsuccessful defibrillation shocks, the early sites of the first and second postshock activations often were located at the same or adjacent electrodes (Table III), implying that there was either a small local reentrant circuit that we did not detect, an automatic focus, or a focus caused by triggered activity (30). After the first few postshock activations, block developed which may have led to reentry and caused VF to regenerate.

The regeneration of VF may occur by the same mechanism that occurs when VF is initiated by stimulation in the vulnerable period of repolarization during sinus rhythm (16). Because of the complexity and rapidity of the activation sequences during $\mathrm{VF}$, at any instant certain regions of the ventricles are repolarizing. Thus a defibrillation shock may always stimulate regions of the ventricles during their vulnerable period, which could cause VF to be regenerated. If a vulnerable period is present in a ventricular region during VF, it probably occurs during the repolarization phase of the action potential of the cells in that region (31). The repolarization phase and hence the vulnerable period should be less than the RR interval during VF, which averaged $105 \pm 16 \mathrm{~ms}$ in this study. Direct intracellular recordings at the onset of VF indicate that the action potential duration is $\sim 50-70 \mathrm{~ms}(32,33)$. Thus, our finding that the preshock intervals at the early sites cluster $\sim 50 \mathrm{~ms}$ (Fig. 15) is consistent with the hypothesis that the region of myocardium at the early site is stimulated during its vulnerable period, causing VF to be reinitiated.
One point against this hypothesis is the presence of the isoelectric window after the shock before VF is regenerated. Premature stimuli whose strength is slightly greater than the fibrillation threshold are thought to cause VF because of dispersion of refractoriness during the vulnerable period (31). This concept predicts that there should be no isoelectric window at the onset of electrically induced VF, because premature stimulation during heterogenous repolarization should excite less refractory portions of the ventricle immediately, and then initiate VF by block and slow conduction to the more refractory portions of the myocardium to create reentry. The strength of defibrillation shocks given in this study expressed as current is about 100 times greater than the current of stimuli at the fibrillation threshold (31). The mechanism of initiation of VF may not be the same for stimuli of low and high strength. Studies are needed of the onset of VF induced by high-energy shocks during the vulnerable period of normal rhythm to see if an isoelectric window is present.

\section{Window width: a determining factor of ventricular defibrillation}

The energy threshold for ventricular defibrillation varies widely (34). Even for energies higher than threshold, there are still a considerable number of failures (35). Therefore, energy is not an excellent predictor of defibrillation outcome. Although different dogs in this study had different energy ranges in which both successful and unsuccessful defibrillations were possible, the ranges of ww associated with successful and unsuccessful defibrillations were similar among all dogs (Fig. 10). The ww was a better predictor of the outcome of defibrillation than was shock energy, voltage, or current.

Thus, ww is a determining factor for ventricular defibrillation. If a shock resulted in a ww of more than $63 \mathrm{~ms}$, successful defibrillation was possible. If the ww exceeded 130 ms defibrillation was always successful. In different dogs, the energy required to produce a certain ww was variable, but the relationship between the ww and the outcome of defibrillation was roughly the same.

\section{Reasons for imperfect correlation of window width and shock strength}

Even with a shock energy as high as $30 \mathrm{~J}$ (three times the defibrillation threshold for that dog), there were still episodes of successful defibrillation in which the ww was $<130 \mathrm{~ms}$. Thus, ww was not predicted well by the energy. The correlation coefficient was only 0.52 . One reason for the relatively poor correlation between ww and shock strength for all dogs taken together was that the slope and intercept of the regression line for each dog varied widely. The correlation coefficient of ww and shock energy for each dog was higher, ranging from 0.53 to 0.95 (Fig. 11). Anatomic, electrophysiologic, and autonomic factors may have been responsible for the dog to dog differences in the regression lines. One point against an anatomic factor is that wide variation still exists in the defibrillation threshold after correction for the heart weight (range 0.031-0.072 J/g heart weight). This does not rule out the possibility that the shape or volume of each heart may influence the current field distribution of the defibrillation shock. Thus an anatomic factor may still be partially responsible for the different regression lines.

Another possible factor that may alter the correlation is the presence of a significant number of inadequate recordings (Fig. 2). Early sites may have been missed in regions with inadequate recordings so that the ww was overestimated. Many inadequate recordings were from electrodes overlying the atria and atrio- 
ventricular sulcus (Figs. 2, 8, 9) or were from isolated electrodes surrounded by good recordings (Fig. $5 \mathrm{~A}$ ) in which the timing of the surrounding electrical activity suggested that an early site in that region was unlikely. Some inadequate recordings occurred in clusters (Figs. $5 A, B$ ), however, so that an early site may have been missed. Nevertheless, experiments with better correlations between the ww and energy did not have a higher percentage of good recordings than those with worse correlations. Thus the inadequate recordings were probably not responsible for the poor correlation between the ww and the energy.

\section{Differences between successful and unsuccessful defibrillation}

Two types of responses were observed after a successful defibrillation shock. The ww separating the two types of responses after a successful shock was $130 \mathrm{~ms}$. This finding confirms that successful defibrillation does not require immediate cessation of all arrhythmic activity after the isoelectric window $(6,36)$. Type B successful defibrillations were characterized by a period of one to three rapid, synchronized activations after the window. In addition, the study demonstrates that unsuccessful shocks transiently slow and synchronize activation before VF resumes. This finding has been reported by Zipes et al. (6) for subthreshold shocks but has not been reported for low level shocks. Even for unsuccessful shocks with energies as low as 1-2 J, we observed slowing and synchronization for up to two postshock activations (Fig. 3). With increasing shock energy, the number of early sites decreased, so that the postshock isochronal maps appeared more organized because fewer activation fronts were present simultaneously (Fig. 6).

Successful type A defibrillation episodes were qualitatively different from unsuccessful defibrillation episodes, having different ww and postshock activation patterns (Figs. 4-6). In contrast, the postshock isochronal maps were similar for successful type B defibrillation episodes and for unsuccessful defibrillation episodes, both of which had a ww $\leq 130 \mathrm{~ms}$. What is the difference between successful type $B$ and unsuccessful defibrillation episodes? We found that for successful type B episodes the mean ww was significantly longer and the mean number of early sites significantly less than for unsuccessful episodes (Table II). Surprisingly, the time for the activation fronts to cross the septum and entire epicardium for the first postshock activation, i.e., the cardiac activation time, was shorter for unsuccessful defibrillation episodes. This time should be related to $(a)$ the number of activation fronts present and hence the number of early sites and (b) the conduction velocity, which should be a function of the state of recovery of the tissue and hence the ww. Indeed, both the ww and the number of early sites correlated negatively with the cardiac activation time for unsuccessful episodes. Thus the nearly twofold greater number of early sites probably made the cardiac activation time for the first postshock activation of unsuccessful defibrillation shorter (Table II). For unsuccessful defibrillation, the cardiac activation time for the second postshock activation was significantly longer than for the first while the number of early sites remained about the same. This increased cardiac activation time could be caused either by a generalized decrease in conduction velocity because of the short $\mathrm{d} 2$ interval or by a change in the activation sequence, one cause of which may have been undirectional block. Both of these factors favor the development of reentry (37-39) and thus may serve as the cause of the postshock regeneration of VF. For successful defi- brillation, on the other hand, the $\mathrm{d} 2$ interval was significantly longer and the increment of cardiac activation time from the first to the second postshock activation was significantly shorter than for unsuccessful episodes (Table II).

The underlying mechanism that gives rise to the observed differences in the number of early sites and the cardiac activation times between these two groups for shocks of the same energy remains unknown. One possibility is that the state of the myocardium at the time of the shock, e.g., the distribution of refractoriness, is different in these two groups. Consequently, the myocardial response to the same sized shock could be different. This difference in response to electrical stimulation is similar to that observed when a stimulus is given in the vulnerable period of normal rhythm, when either VF or multiple extrasystoles can be induced depending on the timing and strength of the stimulus (40). If the postshock regeneration of VF is caused by stimulation during the vulnerable period of a part of the myocardium, as we have discussed, the type B successful defibrillation would serve as the counter part of the multiple extrasystolic responses observed during the vulnerable period of normal rhythm.

Another possible mechanism for the difference between unsuccessful and type B successful episodes may be that the excitation of intracardiac cholinergic and adrenergic nerves by defibrillation shocks (41) may change the effective refractory period of the myocardial cells (42). With increased shock energy the change of the effective refractory period may be more pronounced, or the distribution of the refractoriness may be more uniform, so that the reentry is less likely to occur to reinitiate VF.

\section{Shock field strength and ventricular defibrillation}

The early sites of the first postshock activation were usually located distant from the apical defibrillation electrode in an area not directly depolarized by the low-energy shock given during sinus rhythm (Fig. 12). This suggests that the early sites occurred in the ventricular region in which the field produced by the shock was weakest. To achieve a field sufficient to halt VF in this area, the ventricular tissue adjacent to the defibrillation electrodes probably received much higher amounts of energy than needed to halt VF. Other factors that may have contributed to the location of early sites are the differences of anatomy and autonomic innervation at the base versus the apex. Although it has been shown that stimulation of sympathetic nerve branches can produce localized changes of refractory period (43), it is unclear whether defibrillation shocks can produce different sympathetic tone at the apex versus the base and thus account for the preferential occurrence of early sites at the base after unsuccessful defibrillation.

Early sites after type A defibrillation are not confined to the base but can occur anywhere on the epicardium (Fig. 7). Frequently the early site is on the RV anterior wall near the area of RV breakthrough during normal sinus rhythm, suggesting that the origin of the impulse is supraventricular. However, sinus arrest has been reported to occur after defibrillation (41), and we found that atrial activation occurs later than ventricular activation immediately after the shock. To answer the question of the origin of postshock ventricular excitation for type A recovery, simultaneous recordings from the atrium, the His bundle and the ventricles during the defibrillation shock will be required.

The preferential location of early postshock activation in only a few locations, although showing that the apical and right 
atrial defibrillation electrode configuration is not optimum, indicates that this configuration provides a powerful model for studying the mechanism of successful and unsuccessful defibrillation. The model allows precise measurements and interventions to be performed in the region in which earliest activation appears after the shock. If a way can be found to prevent or delay this early postshock activation, it should be possible to keep VF from being regenerated and to defibrillate with shocks of much lower strength. Because lowering the defibrillation shock strength can decrease myocardial injury (18) as well as decrease energy consumption and prolong the battery life of automatic implantable cardioverter/defibrillators, an understanding of cardiac activation in the immediate postshock period in this model may lead to the improvement of these devices $(44,45)$.

\section{Acknowledgments}

The authors wish to thank Dr. Joseph C. Greenfield, Jr., for his support, Mr. Alton Ledford and Ms. Sharon Bowling for their technical assistance, and Ms. Cloyce Lassiter for her secretarial assistance.

This study was supported in part by training grant HL-07101 and research grants HL-28429 and HL-17670 from the National Heart, Lung and Blood Institute and by a grant from the Fannie E. Ripple Foundation.

\section{References}

1. Tacker, W. A., Jr., and L. A. Geddes. 1980. Electrical Defibrillation. CRC Press, Boca Raton, FL. 1-132.

2. Lepeschkin, E., J. L. Jones, S. Rush, and R. E. Jones. 1978. Local potential gradients as a unifying measure for thresholds of stimulation, standstill, tachyarrhythmia and fibrillation appearing after strong capacitor discharges. Adv. Cardiol. 21:268-278.

3. Jones, J. L., and R. E. Jones. 1982. Determination of safety factor for defibrillator waveforms in cultured heart cells. Am. J. Physiol. 242: H662-H670.

4. Kouwenhoven, W. B., W. R. Milnor, G. G. Knickerbocker, and W. R. Chesnut. 1957. Closed chest defibrillation of the heart. Surgery. 42:550-561.

5. Lown, B. 1976. Electrical reversion of cardiac arrhythmias. $\mathrm{Br}$. Heart J. 29:469-489.

6. Zipes, D. P., J. Fischer, R. M. King, A. D. Nicoll, and W. W. Jolly. 1975. Termination of ventricular fibrillation in dogs by depolarizing a critical amount of myocardium. Am. J. Cardiol. 36:37-44.

7. Smith, W. M., and R. E. Ideker. 1983. Computer techniques for epicardial and endocardial mapping. Prog. Cardiovasc. Dis. 26:15-32.

8. Ideker, R. E., G. H. Bardy, S. J. Worley, L. D. German, and W. M. Smith. 1984. Patterns of activation during ventricular fibrillation. In Tachycardias: Mechanisms, Diagnosis, Treatment. M. E. Josephson, and H. J. J. Wellens, editors. Lea and Febiger, Philadelphia. 519-536.

9. Colavita, P. G., W. M. Smith, P. Wolf, F. R. Bartram, M. Hardage, and R. E. Ideker. 1986. Determination of the effects of internal countershock by direct cardiac recordings during normal rhythm. Am. J. Physiol. In press.

10. Smith, W. M., A. L. Funk, R. E. Ideker, F. R. Bartram, and P. V. Talbert. 1982. A microcomputer-based multichannel data acquisition system for the study of complex arrhythmias. In Proceedings of Computers in Cardiology. IEEE Computer Society, Silver Spring, MD. 131-134.

11. Harrison, L., R. E. Ideker, W. M. Smith, G. L. Klein, J. Kasell, A. G. Wallace, and J. J. Gallagher. 1980. The sock electrode array: a tool for determining global epicardial activation during unstable arrhythmias. Pace. 3:531-540.

12. Worley, S. J., J. L. Swain, P. G. Colavita, W. M. Smith, and R. E. Ideker. 1985. Development of an endocardial-epicardial gradient of activation rate during electrically induced, sustained ventricular fibrillation in dogs. Am. J. Cardiol. 55:813-820.
13. Babbs, C. F. 1978. Effect of pentobarbital anesthesia on ventricular defibrillation threshold in dogs. Am. Heart J. 95:331-337.

14. Amlie, J. P., and T. Owren. 1979. The effect of prolonged pentobarbital anaesthesia on cardiac electrophysiology and inotropy of the dog heart in situ. Acta Pharmacol. Toxicol. 44:264-271.

15. Galino, A. H., and T. B. Davis. 1962. Succinylcholine and cardiac excitability. Anesthesiology. 23:32-40.

16. Wiggers, C. J., and R. Wegria. 1940. Ventricular fibrillation due to single, localized induction and condenser shocks applied during the vulnerable phase of ventricular systole. Am. J. Physiol. 128:500-505.

17. Hoffman, B. F., E. E. Suckling, and C. M. Brooks. 1955. Vulnerability of the dog ventricle and effects of defibrillation. Circ. Res. 3: 147-151.

18. van Vleet, J. F., W. A. Tacker, Jr., L. A. Geddes, and V. J. Ferrans. 1977. Cardiac damage in dogs given multiple transthoracic shocks with a trapezoidal wave-form defibrillator. Am. J. Vet. Res. 38 : 617-626.

19. El-Sherif, N., R. A. Smith, and K. Evans. 1981. Canine ventricular arrhythmias in the late myocardial infarction period. 8. Epicardial mapping of reentrant circuits. Circ. Res. 49:255-265.

20. Bartram, F. R., R. E. Ideker, and W. M. Smith. 1981. A system for the parametric description of the ventricular surface of the heart. Comput. Biomed. Res. 14:533-541.

21. Spiegel, M. R. 1961. "Student's" t distribution. In Schaum's Outline of Theory and Problems of Statistics. M. R. Spiegel, editor. Schaum Publishing Company, New York. 188-190.

22. Ray, A. A. 1982. SAS User's Guide: Statistics. SAS Institute Inc., Cary, NC. 1-514.

23. Ideker, R. E., L. D. German, W. M. Smith, and J. E. Lowe. 1985. Activation rates during human ventricular fibrillation. J. Am. Coll. Cardiol. 5:411. (Abstr.)

24. Perlman, G. 1980. Data analysis programs for the UNIX operating system. Behav. Res. Methods Instrum. 12:554-558.

25. Allessie, M. A., F. I. M. Bonke, and F. J. G. Schopman. 1977. Circus movement in rabbit atrial muscle as a mechanism of tachycardia. III. The "leading circle" concept: a new model of circus movement in cardiac tissue without the involvement of an anatomical obstacle. Circ. Res. 41:9-18.

26. Garrey, W. E. 1914. The nature of fibrillatory contraction of the heart-its relation to tissue mass and form. Am. J. Physiol. 33:397-414.

27. Crampton, R. 1980. Accepted, controversial, and speculative aspects of ventricular defibrillation. Prog. Cardiovasc. Dis. 23:167-186.

28. van Dam, R. T., D. Durrer, J. Strackee, and L. H. van der Tweel. 1956. The excitability cycle of the dogs left ventricle determined by anodal, cathodal, and bipolar stimulation. Circ. Res. 4:196-204.

29. Moore, E. N., and J. F. Spear. 1985. Electrophysiologic studies on the initiation, prevention, and termination of ventricular fibrillation. In Cardiac Electrophysiology and Arrhythmias. D. P. Zipes and J. Jalife, editors. Grune \& Stratton, Inc., Orlando, FL. 315-322.

30. Cranefield, P. F. 1977. Action potentials, afterpotentials, and arrhythmias. Circ. Res. 41:415-423.

31. Han, J. 1973. Ventricular vulnerability to fibrillation. In Cardiac Arrhythmias. L. S. Dreifus and W. Likoff, editors. Grune \& Stratton, Inc., New York. 87-95.

32. West, T. C., E. L. Frederickson, and D. W. Amory. 1959. Single fiber recording of the ventricular response to induced hypothermia in the anesthetized dog correlation with multicellular parameters. Circ. Res. 7:880-888.

33. Downar, E., M. J. Janse, and D. Durrer. 1977. The effect of acute coronary artery occlusion on subepicardial transmembrane potentials in the intact porcine heart. Circulation. 56:217-224.

34. Geddes, L. A., W. A. Tacker, J. Rosborough, A. G. Moore, P. Cabler, M. Bailey, J. D. McCrady, and D. Witzel. 1974. The electrical dose for ventricular defibrillation with electrodes applied directly to the heart. J. Thorac. Cardiovasc. Surg. 68:593-602.

35. Davy, J.-M., E. Fain, P. Dorian, and R. A. Winkle. 1984. Is there a defibrillation "threshold"? Circulation. 70(Suppl. II):406. (Abstr.) 
36. Mower, M. M., M. Mirowski, J. F. Spear, and E. N. Moore. 1974. Patterns of ventricular activity during catheter defibrillation. Circulation. 49:858-861.

37. Arnsdorf, M. F. 1977. Membrane factors in arrhythmogenesis: concepts and definitions. Prog. Cardiovasc. Dis. 19:413-429.

38. Lazzara, R., N. El-Sherif, R. R. Hope, and B. J. Scherlag. 1978. Ventricular arrhythmias and electrophysiological consequences of myocardial ischemia and infarction. Circ. Res. 42:740-749.

39. Josephson, M. E., S. R. Spielman, A. M. Greenspan, and L. N. Horowitz. 1979. Mechanism of ventricular fibrillation in man. Observation based on electrode catheter recordings. Am. J. Cardiol. 44:623631.

40. Hoffman, B. F., E. F. Gorin, F. S. Wax, A. A. Siebens, and C. M. Brooks. 1951. Vulnerability to fibrillation and the ventricularexcitability curve. Am. J. Physiol. 167:88-94.

41. Cobb, F. R., A. G. Wallace, and G. S. Wagner. 1968. Cardiac inotropic and coronary vascular responses to countershock. Circ. Res. 23:731-742.

42. Martins, J. B., and D. P. Zipes. 1980. Effects of sympathetic and vagal nerves on recovery properties of the endocardium and epicardium of the canine left ventricle. Circ. Res. 46:100-110.

43. Kralios, F. A., L. Martin, M. J. Burgess, and K. Millar. 1975. Local ventricular repolarization changes due to sympathetic nerve-branch stimulation. Am. J. Physiol. 228:1621-1626.

44. Mirowski, M., P. R. Reid, M. M. Mower, L. Watkins, Jr., V. L. Gott, J. F. Schauble, A. Langer, M. S. Heilman, S. A. Kolenik, R. D. Fischell, and M. L. Weisfeldt. 1980. Termination of malignant ventricular arrhythmias with an implanted automatic defibrillator in human beings. N. Engl. J. Med. 303:322-324.

45. Griffin, J. C., J. W. Mason, D. L. Ross, and R. V. Calfee. 1981. The treatment of ventricular tachycardia using an automatic tachycardia terminating pacemaker. Pace. 4:582-588. 